\title{
Politiques internationales et maladies non transmissibles : actes manqués et objectifs de développement durable
}

\author{
Non communicable diseases international policies: missed opportunities and sustainable \\ development goals
}

\author{
A. Ly \\ (C) Lavoisier SAS 2015
}

L'intégration de la prise en charge des maladies non transmissibles (MNT) dans les objectifs post 2015 de l'organisation des Nations Unies serait une lueur d'espoir. L'impasse avait été superbement faite sur les MNT lors de la définition des Objectifs du millénaire pour le développement (OMD) en 2000 [1-4]. Une erreur qui n'aurait jamais due être commise si les décisions étaient mieux éclairées par les savoirs scientifiques disponibles à l'époque en dépit des nombreux plaidoyers pro domo et conflictuels qui ont prévalu. En 2000, le compte n'y était pas. En 2015, le compte n'y est toujours pas au moment où les résultats sont examinés et que plusieurs pays ne seront pas au rendez-vous de ces objectifs $[2,4,5]$. Les causes de ces échecs sont multiples et ne pourront pas être toutes abordées ici. De leurs analyses, ouvertes et méthodiques, devraient surgir de nouvelles attitudes, des résolutions innovantes et des concepts renouvelés afin de relever les défis contemporains et futurs.

Parmi ces défis, figurent, en position centrale, les maladies non transmissibles (MNT), premières causes de morbi-mortalité dans le monde. Elles posent bien des questions quant à leur prévention et leur prise en charge [6-8]. En effet, il est imputé à ces pathologies $63 \%$ de la mortalité mondiale soit 36 millions de décès chaque année dont $80 \%$ (29 millions) surviennent dans les pays à revenus faibles ou intermédiaires. Cette mortalité est aussi caractérisée par sa prématurité puisque 14 millions de personnes meurent des suites d'une MNT avant l'âge de 60 ans. La majorité de ces décès prématurés $(86 \%)$ ont lieu dans les pays en développement [8-11]. De surcroît, si les tendances épidémiologiques actuelles perdurent, une augmentation graduelle et dramatique de l'incidence et de la mortalité est prévue. D'ici à 2030, 52 millions de personnes succomberaient annuellement d'une MNT dans le monde $[8,10,11]$. Ces conséquences socio-épidémiologiques des MNT s'aggra-

\footnotetext{
A. Ly $(\bowtie)$

Afrocancer, BP 60751, 75827 Paris cedex 17, France

e-mail : adamaly@gmail.com
}

vent d'une menace progressive sur l'économie mondiale. Ainsi, l'impact économique des maladies non transmissibles pourraient induire des pertes de productivités cumulées énormes qui sont évaluées, pour les deux prochaines décennies (2011-2025), à 7000 milliards dans les pays pauvres et ceux à revenus intermédiaires par l'OMS [8]. En 2010, l'école de santé publique de l'université de Harvard a estimé ces pertes à l'échelle mondiale à 47.000 milliards de dollars US soit 75\% du PIB mondial (63 000 milliards de dollars US) [10].

La réunion de haut niveau sur les MNT organisées sous l'égide de l'Organisation des Nations Unies (ONU) a permis de mettre en exergue cette problématique de santé publique et d'en faire un sujet débattu par les diverses parties concernées et de l'inclure dans l'agenda de la politique internationale. Dans la déclaration politique qui a été adoptée, les états membres reconnaissent que " ces maladies constituent l'un des principaux défis pour le développement au $\mathrm{XXI}^{\mathrm{e}}$ siècle » [12]. Il est établi que les MNT constituent un désastre épidémiologique et économique notamment dans les pays pauvres et brident leur développement économique et social. Ce sont donc des obstacles majeurs pour l'atteinte des OMD. De la même manière, ces écueils, si on n'y prend pas garde, constitueraient une limitation aux incessantes initiatives de réduction de la pauvreté et entraveraient la réalisation des Objectifs de Développement Durable (ODD) qui suivent les OMD et dont le terme est fixé à $2030[6,13,14]$ (Tableau 2).

Ces pays durement impactés seront dans l'incapacité de d'affronter la terrible charge des MNT en raison de l'état asthénique des systèmes de santé et de la faiblesse des investissements publics et privés dans ce secteur malgré les engagements maintes fois pris par les politiques, les institutions régionales et internationales. En Afrique, par exemple, la déclaration d'Abuja, les résolutions sur les MNT et la lutte contre les cancers de l'Union Africaine sont restées sans actions concrètes [15-17]. Ces déficits managériaux répétitifs, le manque de vision d'ensemble, d'anticipation et de prise de responsabilité ad hoc ont comme corollaire une 
Tableau 1 Dépenses militaires en Afrique (d'après la référence 40).

\begin{tabular}{|c|c|c|c|}
\hline & Dépenses en 2014 & Pourcentage $(\%)^{a}$ & (Milliards de dollars) \\
\hline & & 2013-2014 & $2005-2014$ \\
\hline Afrique & 50,2 & $+5,9$ & +91 \\
\hline Afrique du Nord & 20,1 & $+7,6$ & +144 \\
\hline Afrique Subsaharienne & 30,1 & $+4,8$ & +66 \\
\hline \multicolumn{4}{|c|}{ Les changements les plus importants en 2013-2014 } \\
\hline Augmentations & $\%$ & Diminutions & $\%$ \\
\hline Congo, Rep. & +88 & Malawi & 27 \\
\hline Namibie & +47 & Nigeria & 9,3 \\
\hline Zambie & +23 & Ghana & 5,8 \\
\hline Guinée & +22 & Burundi & 5,0 \\
\hline
\end{tabular}

insuffisance des infrastructures techniques et la rareté des ressources humaines qualifiées. Dès lors, les contextes épidémiologiques actuel et prévu devraient susciter des réactions déterminées et durables, surtout politiques, à la mesure de ces enjeux de santé. Les réponses attendues, à l'évidence, sont frappées du sceau de l'urgence. Pour être efficientes, elles devront être innovantes, transversales et en concordance avec la nouvelle donne sanitaire, géoéconomique et sociale. En effet, l'Afrique d'aujourd'hui, est un vaste ensemble de plus d'un milliard d'habitants (16\% de la population mondiale en 2014), plus ouverte sur le reste de la planète et à la mondialisation des échanges [18]. Elle subit de multiples mutations et une pluralité d'influences qui ont des répercussions tout azimut sur son développement. Ainsi, les transitions sont autant épidémiologiques, nutritionnelles, comportementales, sociales, démographiques, qu'environnementales. Des réformes sanitaires structurelles plus que des ajustements conjoncturels sont les besoins actuels et récurrents de nombre de pays. Nier ces complexités et les aspirations des populations est le moyen le plus sûr d'aller vers l'échec. Ces réalités épidémiologiques et sociétales dictent un renouvèlement des approches et des stratégies dans l'élaboration des politiques de santé publique. Les maladies non transmissibles s'accroissent inexorablement alors que les pathologies infectieuses n'affichent pas le grand recul tant espéré. Contre ce double fardeau, les programmes de lutte élargis, cohérents et des interactions pluridisciplinaires feront sens Des solutions sont forcément locales et adaptées en raison des disparités régionales et de la nécessité de coaliser les forces contributives aux niveaux individuel et collectif $[8,10,19-22]$.

Les maladies cardiovasculaires, les cancers, les maladies respiratoires et les diabètes sont les 4 pathologies à qui il est attribué $25 \%$ des décès par MNT. Elles ont en partage certains facteurs de risque tels que le tabagisme, les déséquilibres alimentaires, l'alcoolisme, la sédentarité $[8,11,20]$. Ces affections chroniques, les plus fréquentes, sont les plus ciblées et leurs facteurs de risque relèvent de la santé mondiale. Il n'en demeure pas moins que d'autres types de maladies non transmissibles (allergies, maladies mentales, maladies musculo-squelettiques, etc.) doivent être l'objet d'une attention particulière puisqu'elles sont aussi responsables d'une morbi-mortalité importante à l'échelle de la planète [23]. En conséquence, la question de la définition et du financement des ODD, est posée [12,24,25]. Il d'agira d'abord d'éviter de reconduire des méthodes et recettes éculées, de spécifier des objectifs possibles ou raisonnablement atteignables et d'en délimiter les contours précis. Il faudra des transferts de technologies et de capacités pour enclencher des ruptures organisationnelles et stratégiques à même de guider vers les voies d'un développement soutenable. L'acceptation des ODD repose sur l'implication dès le début de leur élaboration des acteurs de santé, des populations, de leurs représentants et de leurs gouvernements. Les préalables pour leur appropriation sont la bonne gouvernance, l'équité et l'éthique dans leurs réalisations. Les conditions de la réussite de ces changements structurels et de ces évolutions souhaitées demeurent une stabilité politique et sociale durable.

Pourtant, au cours des dernières années, d'importantes avancées ont été faites autant dans la prévention et le diagnostic précoce que dans le traitement des MNT. Cependant, les difficultés cruciales restent l'accès à ces innovations. Les médicaments courants ou essentiels et les infrastructures techniques de base font tragiquement défaut dans nombre de pays en développement. Ainsi, l'exposition à l'insécurité sanitaire concerne 5 milliards d'individus $(75 \%$ de la population mondiale) qui ne disposent soit d'aucune protection sociale soit que d'une couverture maladie incomplète [26,27]. Dans la région subsaharienne, 70 à $80 \%$ de la population n'a pas de protection sociale adéquate [26,28]. Ce qui explique les présentations tardives dans les institutions sanitaires, la gravité et l'état d'avancement des maladies au 
Tableau 2 Les 17 objectifs de développement durable.

\section{Objectif 1}

Eliminer la pauvreté

sous toutes ses formes

et partout dans le monde

\section{Objectif 5}

Réaliser l'égalité des sexes et autonomiser toutes les femmes et les filles

\section{Objectif 9}

Mettre en place

une infrastructure résiliente, promouvoir une industrialisation soutenable qui profite à tous et encourager l'innovation

\section{Objectif 13}

Prendre d'urgence des mesures pour lutter contre les changements climatiques et leurs répercussions

\section{Objectif 17}

Revitaliser le partenariat mondial au service du développement soutenable et renforcer les moyens de ce partenariat

\section{Objectif 2}

Eliminer la faim, assurer

la sécurité alimentaire,

améliorer la nutrition et promouvoir l'agriculture durable

\section{Objectif 6}

Garantir l'accès de tous

à des services

d'approvisionnement

en eau et d'assainissement

et assurer une gestion

durable des services en eau

\section{Objectif 10}

Réduire les inégalités

entre les pays

et en leur sein

\section{Objectif 14}

Conserver et exploiter de manière soutenable les océans, les mers et les ressources marines aux fins du développement

\section{Objectif 3}

Donner aux individus les moyens de vivre une vie saine et promouvoir le bien-être de tous à tous les âges

\section{Objectif 7}

Garantir l'accès de tous à des services énergétiques fiables, durables et modernes à un coût abordable

\section{Objectif 11}

Faire en sorte que les villes et les établissements humains soient ouverts à tous, sûrs, résilients et soutenables

\section{Objectif 15}

Préserver et restaurer

les écosystèmes terrestres, en veillant à les exploiter de façon durable, gérer durablement les forêts, lutter contre la désertification, enrayer et inverser le processus de dégradation des terres et mettre fin à l'appauvrissement de la biodiversité

\section{Objectif 4}

Veiller à ce que tous puissent suivre une éducation de qualité dans des conditions d'équité et promouvoir les opportunités d'apprentissage tout au long de la vie

\section{Objectif 8}

Promouvoir une croissance économique soutenue, partagée et durable, le plein emploi productif et un travail décent pour tous

\section{Objectif 12}

Instaurer des modes de consommation et de production soutenables

\section{Objectif 16}

Promouvoir l'avènement de sociétés pacifiques et ouvertes aux fins du développement durable, assurer à tous l'accès à la justice et mettre en place, à tous les niveaux, des institutions efficaces, responsables et ouvertes moment du diagnostic $[9,11,28]$. Par ailleurs, l'expansion du marché des médicaments contrefaits qui représente $15 \%$ du marché mondial du médicament et $30-40 \%$ des médicaments vendus en Afrique et l'inquiétante augmentation des flux financiers illicites (FFI) sortant de ce continent (50 milliards en 2015) renforcent cette situation délétère d'inaccessibilité aux soins et aux produits de santé [29-32]. Selon le rapport du Groupe de haut niveau sur les FFI en provenance d'Afrique dirigé par Thabo Mbéki, ancien Président d'Afrique du Sud et du Nouveau partenariat pour le développement de l'Afrique (The New Partnership for Africa's
Development (NEPAD), l'arrêt des FFI en 2000 aurait permis au contient africain d'atteindre les OMD en 2016. L'OMD 4, en particulier, qui concerne la réduction de la mortalité materno-infantile aurait pu être réalisé par « la République centrafricaine en 45 ans alors qu'il lui faudrait 218 ans au rythme actuel de progrès, la Mauritanie, 19 ans au lieu de 198 ans, le Swaziland, 27 ans au lieu de 155 ans » [32]. Des conclusions et recommandations de ce rapport, il est souligné que « les ressources que l'Afrique reçoit de ses partenaires extérieurs sous forme d'aide publique au développement n'augmentent pas en raison des difficultés 
financières que connaissent les partenaires, qui au contraire cherchent à réduire ce type de dépense. L'Afrique aura donc besoin de trouver sur le continent lui-même les moyens de financer son développement et de réduire sa dépendance à l'égard de l'aide publique ». Il est, par conséquent, indiscuté que l'engagement des pays de l'Organisation de coopération et de développement économique (OCDE) qui regroupent les pays les plus développés à consacrer $0,7 \%$ de leur Produit National Brut (PNB) à l'aide au développement qui date de 1970, réaffirmé dans plusieurs déclarations et résolutions, devient encore plus illusoire [33]. Rappelons que les états africains eux-mêmes s'étaient engagés en avril 2001 à dédier $15 \%$ de leur PNB à l'amélioration de la santé de leurs populations [15]. A la rencontre de 2013, «Abuja+12», l'écrasante majorité des pays africains n'avait pas respecté cet engagement si essentiel dans le processus de leur développement tant économique que social. Cinq pays, tout au plus, le Rwanda, le Togo, le Madagascar, le Botswana et la Zambie, à cette date, avaient appliqué cette règle commune à plus d'une cinquantaine de pays [34]. La privatisation et la rentabilisation des structures de santé pour pallier l'insuffisance des financements n'ont fait qu'accentuer les inégalités sociales. Le tourisme médical vers l'Europe, l'Amérique, l'Inde ou le Maghreb est le recours de ceux qui, dans ces pays, sont en haut de l'échelle sociale. En Asie et en Amérique latine, les indicateurs de santé sont aussi préoccupants [35,36]. Même dans les pays développés, la fracture sociale s'accroit et les déficits grandissants des systèmes santé restreignent les possibilités d'accès aux médicaments innovants et à certains produits de santé. Par exemple, la lutte pour l'accès à des prix abordables au Sofosbuvir (Sovaldi), le nouveau traitement contre l'hépatite $\mathrm{C}$ des Laboratoires Gilead est emblématique de cette situation de dérives et de crises du sens. Le coût du traitement qui dure environ 12 semaines est estimé à 41000 Euros en France et à 70000 Euros aux États-Unis. Un produit inabordable dont le prix exorbitant est largement discutable et injustifiable financièrement. Un médicament très efficace qui ne pourrait entrer dans l'arsenal thérapeutique et contribuer à la réduction de la mortalité liée à l'hépatite $\mathrm{C}$ qui est de 350000 à 500000 victimes par an sur un total de 130 à 150 millions de personnes infectées dans le monde. En addition, le contrôle de cette affection hépatique par un vaccin curatif ou préventif n'est pas encore réalisable [37].

Ces questions, loin d'être périphériques, nourrissent des incompréhensions et suscitent une demande de corrections politiques de ces inégalités de santé. La couverture sociale universelle est, à ce titre, une réponse pertinente. Plusieurs pays envisagent sa mise en place dans leur système de santé : Sénégal, Rwanda Ghana, Mali, Indonésie, Inde, Turquie, Thailande, Vietnam, etc. [10,26]. En outre, l'augmentation des médicaments génériques et des vaccins prophylactiques à des prix accessibles pourraient permettre aux plus pauvres d'être moins vulnérables et d'avoir un niveau de santé significativement amélioré. Cet élargissement de l'accès aux soins doit se faire d'un point de vue qualitatif. En effet, des dysfonctionnements et des limites dans la qualité des soins sont observés dans les districts de santé en Afrique subsaharienne $[28,38,39]$. Un accès facilité au plus grand nombre ne peut signifier « une institution de santé au rabais » dans laquelle manquent un nombre suffisant de médecins et de spécialités et parfois les accessoires indispensables à la délivrance optimale des soins. Une politique d'inclusion responsable vise à promouvoir à la fois des traitements abordables et de qualité.

La non discrimination est le principe central sur lequel s'appuie la dispensation des soins au service de l'individu et de la collectivité. L'absence de régulation au niveau politique de l'accès aux soins conduit à des inégalités croissantes selon les revenus «santé pour riches » et «santé pour pauvres » et selon la localisation géographique « santé des zones urbaines » et « santé des zones rurales ». Des segmentations qui ont un impact négatif sur la santé générale de la population, l'espérance de vie, les activités sociales et économiques. En réaction à cela, les investissements dans la santé publique doivent être des priorités de développement qui répondent à des besoins vitaux. Toutefois, pour certains pays, les défis à relever se situent ailleurs. Selon l'Institut international de recherche sur la paix de Stockholm (Sipri) les dépenses militaires africaines sont de 50,2 milliards de dollars US en 2014 (soit une augmentation de 5,9\% par rapport à 2013 et de $91 \%$ par rapport à 2005) sur un total mondial qui est évalué à 1776 milliards de dollars US [40] (Tableau 1).

Une deuxième réponse est la transversalité des organisations et des approches associée à la mutualisation des moyens. Les MNT ont des facteurs de risques communs et des stratégies de lutte intégrées peuvent être appliquées $[6,7 \mathrm{a}, 11,20]$. De même, des plateformes techniques modernisées bénéficient aux soins de santé primaires et à tous les types de pathologies. Ce qui démontre, à contrario, que la verticalité à outrance des stratégies est une impasse dans les pays en développement. Ces conditions de travail non exaltantes sont à l'origine du désenchantement des professionnels de santé et expliquent leur migration vers les pays occidentaux créant, de ce fait, un cercle vicieux si dommageable pour les populations [40a,49]. Les reformes indispensables à des changements structurants dans la santé incombent d'abord aux gouvernements locaux à travers les politiques nationales de santé mises en œuvre. Ces fonctions régaliennes des états ne peuvent pas être substituées par un empilement de programmes verticaux financées par diverses aides internationales. La cohésion et la durabilité des choix stratégiques qui font le socle d'une politique de santé conforme aux réalités locales en seraient absentes. Dans l'autre sens, la finalité de la coopération internationale 
Nord - Sud est de favoriser l'émergence des compétences locales à travers le renforcement des capacités, les transferts de technologies et d'aider à la définition et à l'application des priorités de santé gouvernementales [24,25a,25b].

Il s'agit d'aider sans remplacer ni déresponsabiliser. Les aides sont encore vitales pour les pays à ressources limitées et ont contribué de façon essentielle à la construction des systèmes de santé et ce depuis les indépendances notamment pour les nations subsahariennes [19,21,22,24,38]. Par contre, les effets pervers courants en sont la transformation des ressources humaines locales en collaborateurs plus qu'en acteurs du développement de la santé nationale [41-43]. Les responsabilités respectives sont donc à questionner. La dépendance vis-à-vis des financeurs et à l'expertise extérieure fausse souvent la définition des priorités de santé publique et introduit un fonctionnement tutélaire qui ne favorise pas des trajectoires de développement propres [4143, 43a,44]. Les politiques de santé manquent d'envergures sous-régionale, régionale ou continentale alors que l'échelon de pertinence se trouve à ces niveaux d'organisations. En effet, l'unité culturelle des peuples, la génétique des populations, les groupes ethniques, les proximités géographiques plaident logiquement pour une prise en considération de ces ensembles et sous-ensembles sociologiques et pour une coopération entre les pays du sud [13,17,20-22]. De telles approches de santé publique permettent d'ouvrir des pistes de réflexion nouvelles. Mais, ces changements alternatifs seront lents et ardus. Une des raisons est que les frontières habituelles sont maintenues fermées par des conflits d'intérêts personnels ou catégoriels, les divisions géopolitiques en zones d'influences et les cloisonnements selon les langues officielles des pays. Ces déterminants complexes (historiques et conflictuels) sous-tendent les problématiques de santé dans les pays pauvres. Ils doivent être appréhendés par qui veut y démêler l'écheveau des enjeux de politiques nationales et internationales de santé.

Seulement, de l'histoire de l'humanité, les connaissances scientifiques d'aujourd'hui et les possibilités thérapeutiques et préventives actuelles n'ont jamais été aussi vastes. Des millions de vies à travers la planète peuvent être préservées. Nombre de MNT sont évitables, curables ou contrôlables. Ces constations tendent à montrer que les obstacles sont moins techniques et médico-scientifiques que politiques et socioéconomiques. Comme pour les risques infectieux graves (maladie à virus Ebola, syndrome d'immunodéficience acquise (SIDA), syndrome respiratoire aigue sévère (SRAS)), les politiques publiques devraient faire en sorte que l'accès aux soins soit fonction des besoins de santé et non déterminés par le seul pouvoir d'achat. Le droit à la santé est ainsi l'un des droits humains les plus fondamentaux et admis comme tel dans la déclaration universelle des droits de l'Homme de 1948. Il reconnait à tout individu le « droit à un niveau de vie suffisant pour assurer sa santé, son bien-être et ceux de sa famille » [45]. Ce droit est réaffirmé par l'Organisation Mondiale de la Santé (OMS) dans sa constitution [46]. Néanmoins, chaque année dans le monde, les dépenses de santé font basculer 150 millions de personnes dans des difficultés financières dont les $2 / 3$ se retrouvent en dessous du seuil de pauvreté $[26,27]$. Ces tensions autour des produits de santé et de leurs accès ne s'expliquent que par des arguments d'ordres stratégiques et financiers. Pendant que l'OMS admet que la santé est un droit humain, l'Organisation Mondiale du Commerce $(\mathrm{OMC})$ règlemente à travers les brevets l'accessibilité aux médicaments. Or, le brevet est devenu un outil manipulable et manipulé à des fins autres que celles de sa création $[47,48]$. En réalité, les maladies transmissibles ne sont chroniques que dans les pays développés où leur contrôle est désormais un acquis. Ailleurs, dans le reste du monde, elles sont rapidement mortelles avec des complications souvent graves et des comorbidités causant des souffrances inhumaines [19,28,49-53]. Prenant le risque de la naïveté, il est permis de penser qu'un monde plus paisible et plus stable ne peut laisser sur le bas côté de l'autoroute de l'opulence une fraction importante de l'humanité vivant sous le seuil de pauvreté ou subissant stoïquement les conséquences désastreuses de décisions prises par d'autres. L'unanimité sur l'universalité du droit à la santé doit s'accompagner de mesures effectives au-delà des déclarations de bonnes intentions. Enfin, la complexité des aspects sanitaires tient à ces quadruples dimensions politique, économique, sociale et culturelle. La prise en charge des maladies non transmissibles au niveau national et transnational est assurément un objectif de développement durable prioritaire compte tenu de leur poids majoritaire dans la mortalité mondiale et des funestes impacts socio-économiques induits. Elle est aussi le gage d'une politique internationale responsable, éthique et solidaire dans la réduction des risques de santé.

\section{Références}

1. ONU (2000) Les objectifs du millénaire pour le développement. Déclaration du millénaire sommet du millénaire, New York, septembre http://www.un.org/fr/millenniumgoals/

2. UN (2014) Objectifs du Millénaire pour le développement Rapport 2014, Nations Unies New York

3. Alleyne G, Binagwaho A, Haines A, et al (2013) Embedding noncommunicable diseases in the post-2015 development agenda. Lancet 381(9866):566-74

4. Fehling M, Nelson BD, Venkatapuram S (2013) Limitations of the Millennium Development Goals: a literature review. Glob Public Health 8(10):1109-22

5. UN (2015) Un bilan mitigé pour les objectifs du Millénaire liés à la santé, selon l'OMS http://www.un.org/sustainabledevelopment/ fr/2015/05/18/2436/ Consulté le 2 juin 2015

6. Mitchell E, Daniels Jr, Thomas E, et al (2014) The emerging global health crisis. Non Communicable Diseases in Low- and Middle-Income Countries, Independent Task Force Report No. 72, New-York, Council on Foreign Relations http://cyber. 
law.harvard.edu/people/tfisher/Health/CFR_NCDCrisis_2014.pdf Consulté le 12 juin 2015

7. WHO (2011) Scaling up action against non communicable diseases: How much will it cost? Geneva, World Health Organization

7. a.Muka T, Imo D, Jaspers L, et al (2015) The global impact of non-communicable diseases on healthcare spending and national income: a systematic review. Eur J Epidemiol 30(4):251-77

8. OMS (2014) Rapport sur la situation mondiale des maladies non transmissibles 2014 «Atteindre les neuf cibles mondiales : une responsabilité partagée » http://apps.who.int/iris/bitstream/10665/ 149294/1/WHO NMH NVI 15.1 fre.pdf Consulté le 8 juin 2015

9. OMS (2015) Statistiques sanitaires mondiales 2014, Genève, OMS http://www.who.int/gho/publications/world_health_statistics/2014/ fr/ Consulté le 02 Juin 2015

10. Bloom DE, Cafiero ET, Jané-Llopis E, et al (2011). The Global Economic Burden of Non communicable Diseases. Geneva: World Economic Forum and Harvard School of Public Health. http://www.weforum.org/reports/global-economic-burden-noncommunicable-diseases Consulté le 11 Juin 2015

11. Norheim OF, Jha P, Admasu K, et al (2015) Avoiding 40\% of the premature deaths in each country, 2010-30: review of national mortality trends to help quantify the UN sustainable development goal for health. Lancet 385(9964):239-52

12. UN (2011) Réunion de haut niveau sur les maladies non transmissibles : l'assemblée adopte une déclaration politique qui engage les états membres à s'aligner sur les directives de l'OMS http://www. un.org/press/fr/2011/AG11138.doc.htm Consulté le 04 Juin 2015

13. Brolan CE, Lee S, Kim D, et al (2014) Back to the future: what would the post-2015 global development goals look like if we replicated methods used to construct the Millennium Development Goals? Global Health 10:19

14. Shah E, Liam S (2008) Non-communicable diseases in low and middle-income countries: a priority or a distraction? Int J Epidemiol 34:961-6

15. OAU (2001) Abuja declaration on HIV / AIDS, Tuberculosis and other related infectious diseases, Abuja, Organization of African Unity (OAU) http://www.un.org/ga/aids/pdf/abuja_declaration. pdf Consulté le 02 Juin 2015

16. Union Africaine (2013) Rapport sur le cancer en Afrique. Conférence des ministres de la santé de l'Union Africaine (CAMH6) Sixième session ordinaire, 22-26 avril 2013, Addis-Abeba (Éthiopie), CAMH/Exp/6(VI) ii, $23 \mathrm{p}$

17. UA (2013) Impact des maladies non transmissibles (MNT) et des maladies tropicales négligées (MTN) sur le développement de l'Afrique. Sixième Conférence des ministres de la santé de 1'Union Africaine 22-26 Avril 2013 Addis-Abeba (Ethiopie) $\mathrm{CAMH} / \mathrm{Exp} / 6(\mathrm{VI})$

18. UN (2014) Population growth and universal access to reproductive health[REMOVED HYPERLINK FIELD]http://www.un.org/en/ development/desa/population/publications/pdf/popfacts/PopFacts 2014-6.pdf Consulté le 05Juin 2015

19. Remais JV, Zeng G, Li G, et al (2013) Convergence of noncommunicable and infectious diseases in low- and middle-income countries. Int J Epidemiol 42(1):221-7

20. Ly A (2012) Cancers et autres maladies non transmissibles : vers une approche intégrée de santé publique J Afr Cancer 4:137-9

21. Mc Carthy M, Maher D, Ly A, et al (2010) Developing the agenda for European Union collaboration on non communicable diseases research in Sub-Saharan Africa. Health Research Policy and Systems, 8:13 doi: 10.1186/1478-4505-8-13

22. Sylla BS, Wild[REMOVED HYPERLINK FIELD] CP (2012) Cancer burden in Africa in 2030: Invest today and save tomorrow. J Afr Cancer 4:1-2

23. Lopez AD, Williams TN, Levin A, et al (2014) Remembering the forgotten non-communicable diseases. BMC Med 12(1):200. doi: 10.1186/s12916-014-0200-8
24. Hill PS, Buse K, Brolan CE, et al (2014) How can health remain central post-2015 in a sustainable development paradigm? Global Health 10:18

25. Banque mondiale (2014) Objectifs du Millénaire pour le développement : la vision de la Banque mondiale pour l'après-2015 http://www.banquemondiale.org/fr/news/feature/2014/03/13/ world-bank-presents-views-on-post-2015-framework-for-mdgs Consulté le 11 Juin 2015

25. a.OCDE (2010) Aid for Better Health, What are we learning about what Works and what we still have to do? An Interim report from the Task Team on Health as a tracer sector, Paris, OCDE,78 $\mathrm{p}$.

25. b.Balabanova D, McKee M, Mills A, et al (2010) « What Can Global Health Institutions Do to Help Strengthen Health Systems in Low Income Countries? », Health Research Policy and Systems, 8 (22) doi: 10.1186/1478-4505-8-22

26. BIT (2011) Socle de protection sociale pour une mondialisation juste et inclusive. Rapport du groupe consultatif présidé par Michelle Bachelet mis en place par le BIT avec la collaboration de l'OMS, Genève, Bureau international du Travail

27. OMS (2010) Le financement des systèmes de santé : le chemin vers la couverture universelle. Rapport sur la santé dans le monde, Genève, éditions OMS

28. de-Graft Aikins A, Kushitor M, Koram K, Gyamfi S, et al (2014) Chronic non-communicable diseases and the challenge of universal health coverage: insights from community-based cardiovascular disease research in urban poor communities in Accra, Ghana. BMC Public Health 14 Suppl 2:S3. doi: 10.1186/1471-2458-14$\mathrm{S} 2-\mathrm{S} 3$

29. OMS (2010) La menace croissante des contrefaçons de médicaments Bulletin de l'Organisation mondiale de la Santé Volume 88 pp .241-320 http://www.who.int/bulletin/volumes/88/4/10-020410/ fr/ Consulté le 11 Juin 2015

30. Albert I. Wertheimer, Perry G. Wang (2012) Counterfeit Medicines Volume I : Policy, economics and Countermeasures, ILM Publications

31. Institut de recherche contre le faux médicament (IRCAM) (2013) rapport sur les contrefaçons de médicaments http://www. iracm.com/wp-content/uploads/2013/09/A-Rapport-Etude_IRACM Contrefacon-de-Medicaments-et-Organisations-Criminelles_FR FINAL-copie-2.pdf Consulté le 11 Juin 2015

32. Nations-Unies et Union Africaine (2015) Rapport du Groupe de haut niveau sur les flux financiers illicites en provenance d'Afrique. Rapport établi à la demande de la Conférence conjointe UA/CEA des ministres des finances, de la planification et du développement économique Addis- Abeba, CEA.

33. OCDE (2015) L'objectif de $0.7 \%$ APD/PNB - un historique http://www.oecd.org/fr/cad/stats/45539389.pdf Consulté le 08 Juin 2015

34. WHO (2011) Abuja declaration: Ten years on Geneva, World Health Organization

35. David E.Bloom, Elizabeth T, Cafiero Mark E. McGovern, et al (2013). The economic impact of non communicable disease in China and India: estimates, projections, and comparisons. NBER Working Paper No. 19335. Cambridge, MA: National Bureau of Economic Research

36. Crabtree-Ramírez B, Del Río C, Grinsztejn B, et al (2014) HIV and noncommunicable diseases (NCDs) in Latin America: a call for an integrated and comprehensive response. J Acquir Immune Defic Syndr 67 Suppl 1:S96-8. doi: 10.1097/QAI.0000000000000261

37. OMS (2014) Hépatite C Aide-mémoire $N^{\circ} 164$ http://www.who. int/mediacentre/factsheets/fs164/fr/ Consulté le 17 Mai 2015

38. Union Africaine (2007) Renforcer les systèmes de santé pour l'équité et le développement en Afrique, Projet Stratégie africaine de la santé : 2007 à 2015 CAMH/MIN/5(III). 3ème session 
ordinaire de la conférence ses ministres de la santé de l'Union Africaine, Johannesburg (Afrique du Sud) 9 - 13 Avril 2007

39. J.P.Olivier de Sardan, V. Ridde (eds) (2014) Une politique publique de santé et ses contradictions. La gratuité des soins au Burkina Faso, au Mali et au Niger, Paris, Karthala

40. Sam Perlo-Freeman, Aude Fleurant, Pieter D. Wezeman and Siemon T. Wezeman (2015)Trends in world military expenditure, 2014, Stockholm International Peace Research Institute (SIPRI) Fact Sheet April 2015, http://books.sipri.org/files/FS/SIPRIFS 1504.pdf Consulté le 12 Juin 2015

40. a.Eastwood JB, Conroy RE, Naicker S, et al (2005) Loss of health professionals from sub-Saharan Africa: the pivotal role of the UK. The Lancet, 365(9474) : 1893-1900

41. Gérard Etienne, Wagner Anne-Catherine (2015) Elites au Nord, élites au Sud: des savoirs en concurrence? Cahiers de la recherche sur l'éducation et les savoirs, 14, p. 7-24

42. Jean François Mattei (2014) L'humanitaire à l'épreuve de l'éthique, Paris, Editions " les liens qui libèrent»

43. Rey J.L, Livinec B, Milleliri J.M. (2013) La santé, dernier champ de la tutelle étrangère en Afrique ? Médecine et Santé Tropicales $23: 16-17$

43. a.Dominique Kerouedan (2013) Globaliser n'est pas sans risques pour les populations les plus pauvres du monde, Émergence de la « Global Health » en Afrique subsaharienne, Revue Tiers Monde $\mathrm{N}^{\circ} 215$ pp111-127

44. Gentilini M. (2013) Coopération. Med Sante Trop $23: 357-8$.

45. UN (1948) Universal Declaration of Human Rights. Geneva, United Nations

46. WHO (1946) Constitution of the World Health Organization. New York, World Health Organization
47. Organisation Mondiale de la Propriété Intellectuelle (OMPI) (2011), Rapport sur la propriété intellectuelle dans le monde. Le nouveau visage de l'innovation, Série Économie et statistiques de l'OMPI. http://www.wipo.int/edocs/pubdocs/fr/intproperty/944/ wipo_pub_944_2011.pdf Consulté le 15 Mai 2015

48. Bogers M, Bekkers R, Granstrand O (2012) Intellectual Property and Licensing Strategies in Open Collaborative Innovation in De Pablos Heredero C. et López D., (Eds), Open Innovation in Firms and Public Administrations: Technologies for Value Creation, Hershey IGI Global, 37-58

49. Jaspers L, Colpani V, Chaker L, et al (2015) The global impact of non-communicable diseases on households and impoverishment: a systematic review. Eur J Epidemiol 30(3):163-88

50. Bloomfield GS, Khazanie P, Morris A, et al (2014) HIV and non communicable cardiovascular and pulmonary diseases in low- and middle-income countries in the ART era: what we know and best directions for future research.J Acquir Immune Defic Syndr 67 Suppl 1:S40-53. doi: 10.1097/QAI.0000000000000257.

51. Vineis P, Stringhini S, Porta M (2014) The environmental roots of non-communicable diseases (NCDs) and the epigenetic impacts of globalization. Environ Res 133:424-30

52. Kengne AP, June-Rose McHiza Z, Amoah AG, et al (2013) Cardiovascular diseases and diabetes as economic and developmental challenges in Africa. Prog Cardiovasc Dis 56(3):302-13

53. Sasco Annie J, Jaquet, Antoine; Dabis, François; Malvy, Denis (2011) Cancer and HIV in Sub-Saharan Africa: Not Exactly as in the North Journal of Acquired Immune Deficiency Syndromes 56:42. DOI: 10.1097/01.qai.0000397297.18174.4d 